\title{
Die Weiterentwicklung der Europäischen Union: Vertiefung versus Erweiterung?
}

\author{
Anne Faber*
}

\begin{abstract}
,Vertiefung ‘ und ,Erweiterung '1 sind seit Anbeginn des Integrationsprozesses die beiden zentralen Antriebskräfte und Perspektiven für die Weiterentwicklung der Europäischen Gemeinschaft/Europäischen Union, hinter denen gleichzeitig immer auch spezifische Leitbilder $^{2}$ für die zukünftige Konstruktion Europas wie auch für die tagespolitische Agenda standen. ${ }^{3}$ Im Zuge der großen Erweiterungsrunde um zehn neue Mitgliedstaaten im Mai 2004 wurde jedoch in der wissenschaftlichen wie auch in der politischen Debatte immer häufiger diskutiert, ob sich diese beiden Leitbilder der Integrationsentwicklung nicht zu zwei einander diametral entgegengesetzten Polen entwickelt hätten und die Europäischen Union in einem fortdauernden Dilemma gefangen hielten: „Never before the tension between widening and deepening has become $[\ldots]$ so obvious. “4

Der folgende Beitrag untersucht die Strategien, die der Europäischen Union im Spannungsfeld zwischen Vertiefung und Erweiterung gegenwärtig zur Verfügung stehen. Nach einem kurzen konzeptionellen Aufriss der grundsätzlich denkbaren Strategien und Antworten wird die begrenzte Zahl der politisch mehrheitsfähigen Strategien vorgestellt, die dann im dritten Teil diskutiert werden. Ziel des Beitrages ist es zu zeigen, dass aus dieser begrenzten Anzahl der politisch durchsetzbaren Strategien gegenwärtig keine auch nur ansatzweise den Herausforderungen und Fragen gerecht wird, die sich der neuen EU-27 stellen. Anstelle der traditionellen Strategien, so das Argument, muss die Europäische Union in den kommenden Jahren daher zu neuen, deutlich differenzierteren Konzepten und Antworten in der Vertiefungs- und Erweiterungsfrage finden. Diese Antworten werden sich gleichzeitig in ein
\end{abstract}

* Dr. Anne Faber, wissenschaftliche Mitarbeiterin, Jean Monnet Lehrstuhl, Forschungsinstitut für Politische Wissenschaft und Europäische Fragen, Universität zu Köln.

Ich danke Christina Zuber für ihre Mitarbeit an diesem Beitrag sowie den Kolleginnen und Kollegen am Lehrstuhl und den Gutachtern für ihre konstruktiven Anmerkungen.

1 Unter, Vertiefung ' wird dabei üblicherweise die Ausweitung der Politikformulierungskompetenzen der Europäischen Union auf neue Politikbereiche sowie die Stärkung der Kompetenzen der europäischen Institutionen, das heißt ein Prozess der allmählichen und formalen vertikalen Institutionalisierung, verstanden, während der Begriff der ,Erweiterung' die geografische Ausweitung des Gültigkeitsbereichs von EU-Recht und europäischer Politik bezeichnet, das heißt den Beitritt neuer Mitgliedstaaten oder den Prozess der schrittweisen, formalen horizontalen Institutionalisierung. Vgl. Frank Schimmelfennig/Ulrich Sedelmeier: Theorizing EU enlargement: research focus, hypotheses, and the state of research, in: Journal of European Public Policy 4/ 2002, S. 500-528, hier S. 502.

2 Vgl. zur Leitbild-Debatte Heinrich Schneider: Europäische Integration: die Leitbilder und die Politik, in: Michael Kreile: Die Integration Europas, Opladen 1992, S. 3-35; Heinrich Schneider: Deutsche Europapolitik: Leitbilder in der Perspektive - Eine vorbereitende Skizze, in: Mathias Jopp/Uwe Schmalz/Heinrich Schneider (Hrsg.): Eine neue deutsche Europapolitik? Rahmenbedingungen- Problemfelder- Optionen, Bonn 2002, S. 69131; Roland Hierzinger (Hrsg.): Europäische Leitbilder: Festschrift für Heinrich Schneider, Baden-Baden 2001; Hartmut Marhold (Hrsg.): Die neue Europadebatte - Leitbilder für das Europa der Zukunft, Bonn 2001.

3 So auch Fridrichs, Mihov und Popova: ,Both in the theory and in the practice of European integration, substantive broadening, geographical widening and institutional deepening have been, and are still, the nuts and bolts of permanent reform initiated in the mid-1960s“. Jörg Fridrichs/Jordan Mihov/Maria Popova: Synergies and Tradeoffs in International Cooperation: Broadening, Widening, and Deepening, European integration online papers 13/2005, S. 1.

4 Wolfgang Weiß: Eastern Enlargement and European Constitutionalisation, Queen's Papers on Europeanisation 1/2005, S. 9 . 
stimmiges Gesamtkonzept integrieren und den Bürgerinnen und Bürgern der Union vermitteln lassen müssen, um der wachsenden Erweiterungsskepsis in den alten wie den neuen Mitgliedstaaten begegnen zu können: „Die EU wird eine neue Balance zwischen Erweiterung und Aufnahmefähigkeit finden und dies gegenüber den Bürgerinnen und Bürgern in der Union und in den Kandidatenländern kommunizieren müssen. "5

Die deutsche Ratspräsidentschaft sollte es sich daher auch zur Aufgabe machen, regelmäBige Konsultationen zur Entwicklung einer zukunftsfähigen gemeinsamen Strategie der Europäischen Union zwischen Erweiterung und Vertiefung einzuleiten. Ohne grundsätzliche Beratungen über die zukünftige strategische Ausrichtung und Weiterentwicklung des europäischen Integrationsprojektes werden auch Entscheidungen über konkrete politische Projekte und neue Erweiterungsrunden in der EU-27 zunehmend schwerer zu treffen sein.

\section{Leitbilder und Strategien für die Weiterentwicklung der Europäischen Union: Kon- zeptioneller Aufriss}

Gegenwärtig stellen sich eine Reihe von dringlichen Grundsatzfragen hinsichtlich der zukünftigen Ausgestaltung der Europäischen Union: Ist politische Integration, das heißt ein weiterer Ausbau der institutionellen Struktur der Europäischen Union und die Ausdehnung ihrer Aufgabenbereiche auf neue Politikfelder, ein Projekt, das nur mit einer begrenzten Anzahl von Mitgliedstaaten erreichbar ist (beziehungsweise erreichbar gewesen wäre)? Wird sich die Europäische Union unbegrenzt erweitern (müssen)? Und wie wird eine sich immer wieder erweiternde Europäische Union institutionell und konstitutionell so gestaltet werden können, dass sie handlungsfähig bleibt?

Die politikwissenschaftliche Debatte hat nach wie vor kaum Analysen zu den systematischen Auswirkungen bisheriger Erweiterungen auf die Vertiefung des Integrationsprozesses und umgekehrt zu den Effekten von Vertiefung auf Erweiterungsvorhaben vorgelegt, die in der Lage wären, zur Beantwortung dieser Fragen beizutragen. ${ }^{6}$

Die konzeptionell denkbaren politischen Strategien zwischen Vertiefung und Erweiterung lassen sich in neun Idealtypen gruppieren. Sie spiegeln gleichzeitig die europapolitischen Leitbilder und Strategien der Regierungen der Mitgliedstaaten als den in dieser Frage letztlich entscheidenden Akteuren wider, die sich deutlich voneinander unterscheiden:

5 Michael Dauderstädt/Barbara Lippert/Andreas Maurer: Die deutsche EU-Ratspräsidentschaft 2007: Hohe Erwartungen bei engen Spielräumen, Internationale Politikanalyse Friedrich Ebert Stiftung 11/2006, S. 28.

6 Vgl. zu diesem , blinden Fleck' in der politikwissenschaftlichen Debatte ausführlicher Anne Faber: Theoretical Approaches to EU Deepening and Widening: A Multi-disciplinary Overview and Some Tentative (Hypo)theses, S. 3-6, abrufbar unter: http://www.eu-consent.net/content.asp?contentid=1247 (letzter Zugriff: 06.02. 2007); Bernard Steunenberg: Enlargement and reform in the European Union, in: Bernard Steunenberg (Hrsg.): Widening the European Union. The Politics of institutional change and reform, London/New York 2002, S. 3-20. Eine Ausnahme ist Barbara Lippert: Enlargement: the political and constitutional implications, in: Fergus Carr/ Andrew Massey (Hrsg.): Public Policy and the new European Agendas, Cheltenham, UK/Northampton, MA, 2006 S. 99-131. 
Politische Strategien zwischen Vertiefung und Erweiterung: Neun Idealtypen

\begin{tabular}{|l|l|l|l|}
\hline Erweitern & \multicolumn{1}{|c|}{ Vertiefen } & \multicolumn{1}{|c|}{ zweitrangig } & \multicolumn{1}{|c|}{ nie } \\
\hline erstrangig & $\begin{array}{l}\text { Gleichzeitig erweitern } \\
\text { und vertiefen }\end{array}$ & $\begin{array}{l}\text { Zunächst erweitern, um } \\
\text { dann zu vertiefen }\end{array}$ & $\begin{array}{l}\text { Erweitern, nicht aber } \\
\text { vertiefen }\end{array}$ \\
\hline zweitrangig & $\begin{array}{l}\text { Zunächst vertiefen, um } \\
\text { dann zu erweitern }\end{array}$ & $\begin{array}{l}\text { Nach einer Phase der } \\
\text { Konsolidierung auf der } \\
\text { Basis des Status quo } \\
\text { gleichzeitig erweitern } \\
\text { und vertiefen }\end{array}$ & $\begin{array}{l}\text { Nach einer Phase der } \\
\text { Konsolidierung auf der } \\
\text { Basis des Status quo } \\
\text { weitere Erweiterungs- } \\
\text { projekte vorsehen, } \\
\text { nicht aber vertiefen }\end{array}$ \\
\hline nie & $\begin{array}{l}\text { Vertiefen, nicht aber er- } \\
\text { weitern }\end{array}$ & $\begin{array}{l}\text { Nach einer Phase der } \\
\text { Konsolidierung auf der } \\
\text { Basis des Status quo } \\
\text { weitere Vertiefungspro- } \\
\text { jekte vorsehen, nicht } \\
\text { aber erweitern }\end{array}$ & $\begin{array}{l}\text { Weder erweitern noch } \\
\text { vertiefen, sondern eine } \\
\text { langfristige Phase der } \\
\text { Konsolidierung auf der } \\
\text { Basis des status quo } \\
\text { vorsehen }\end{array}$ \\
\hline
\end{tabular}

Aus diesen neun idealtypischen Strategien lassen sich drei ${ }^{7}$ hervorheben, die in der europapolitischen Debatte um die Weiterentwicklung des europäischen Einigungsprojektes weiterhin - wenn auch mit gewissen Akzentverschiebungen - diskussionsleitend sind:

- Zunächst vertiefen, um dann zu erweitern

- Zunächst erweitern, um dann zu vertiefen

- Gleichzeitig erweitern und vertiefen

Sie werden im Folgenden einzeln vorgestellt, um zu verdeutlichen, dass keine dieser ,klassischen' Strategien in der Lage sein wird, den zukünftigen Herausforderungen europäischer Integration gerecht zu werden.

\section{Vertiefung und Erweiterung in der politischen Diskussion: Alte Strategien für eine neue Europäische Union?}

Die drei oben genannten Konzepte und Strategien haben eine lange Tradition in der Geschichte des europäischen Einigungsprozesses. Dennoch lassen sich seit Beginn der 1990er Jahre, die zunehmend von der Perspektive der Großerweiterung geprägt waren, vermehrt Akzentverschiebungen innerhalb und zwischen den Konzepten feststellen. Diese Akzentverschiebungen weisen auf die Fragen hin, mit denen die Europäische Union gegenwärtig konfrontiert ist.

\section{Zunächst vertiefen, um dann zu erweitern}

Diese Strategie geht davon aus, dass nur eine institutionell und politisch gefestigte Union in der Lage ist, neue Mitglieder aufzunehmen, ohne den erreichten Integrationsgrad zu gefährden. Daher, so das Argument, sollten Reform- und Vertiefungsprojekte stets Vorrang

7 Vgl. auch Barbara Lippert/Wolfgang Wessels: Erweiterungskonzepte und Erweiterungsmöglichkeiten, in: Cord Jakobeit/ Alparaslan Yenal (Hrsg.): Gesamteuropa. Analysen, Probleme und Entwicklungsperspektiven, Schriftenreihe der Bundeszentrale für politische Bildung, Band 317, Studien zur Geschichte und Politik, Bonn 1993, S. 439-457. 
vor Erweiterungen haben. Zugespitzt formuliert kann diese Position daher auch als ,Vertiefen, nicht aber erweitern " verstanden werden. Allerdings wird diese radikalere Position gegenwärtig von keinem Mitgliedstaat ernsthaft vertreten, da sie sowohl der Selbstwahrnehmung der Europäischen Union als auch den bereits gemeinsam getroffenen, verbindlichen Zusagen an die neuen Beitrittskandidaten Türkei und Kroatien (Beginn der Beitrittsverhandlungen je 3. Oktober 2005), sowie Mazedonien (Kandidatenstatus seit dem 16. Dezember 2005) widerspricht.

In der Form ,Zunächst vertiefen, um dann zu erweitern ' hat die Strategie seit der Erweiterungsrunde 2004 in der politischen Debatte jedoch deutlich an Unterstützung gewonnen, was auf eine - temporäre oder permanente - ,Erweiterungsmüdigkeit' in der Union hinweist. Die Akzentverschiebung drückt sich nicht zuletzt in den neuen Prinzipien aus, die die Europäische Union für ihre zukünftige Erweiterungspolitik definiert hat:

- Konsolidierung des erreichten Integrationsniveaus,

- Konditionalität weiterer Beitrittsverhandlungen,

- Kommunikation der Vorgehensweise der Europäischen Union in den Mitgliedstaaten.

Dabei hält die Europäische Kommission explizit fest: „Die Konsolidierung der Erweiterungsagenda der EU bedeutet, dass diese zurückhaltend ist, wenn es um die Übernahme neuer Verpflichtungen geht. “68

Vertreten wird dieses Konzept vor allem von Frankreich, Österreich und Dänemark, die allesamt eine genauere Definition der Aufnahmekapazität und der Grenzen der Europäischen Union wünschen. ${ }^{9}$ Diese Mitgliedstaaten fürchten nicht nur um den geografischen, sondern auch um den kulturellen Zusammenhalt der Union und damit um den weiteren Aufbau einer europäischen Basisidentität. Einige der südeuropäischen Staaten fürchten im Zuge neuerlicher Erweiterungsrunden darüber hinaus um ihre Anteile an Fördermitteln und -programmen. Eine sich permanent erweiternde Union würde, so das Argument, zu einer, Überdehnung ' der institutionellen Struktur und des politischen und wirtschaftlichen Zusammenhaltes führen. Die Entwicklungsszenarien, die hieraus resultieren könnten, wären eine europäische Freihandelszone oder - günstigstenfalls - eine Wirtschaftsunion mit partieller Währungsintegration.

Die deutsche Bundeskanzlerin und derzeitige EU-Ratsvorsitzende, Angela Merkel, hat sich unlängst in zwei Reden für eine vorrangige Vertiefung ausgesprochen: „Es gibt Mitgliedstaaten, die prioritär auf die Vertiefung der Integration setzen, und es gibt Mitgliedstaaten, die sehr stark auf eine Ausdehnung setzen. Manch einer glaubt, dies alles könne parallel geschafft werden; ich glaube nicht, dass das klappt. Und deshalb ist aus meiner Sicht die Aussage richtig, dass wir auf absehbare Zeit [...] keine neuen Zusagen machen können, was Mitgliedschaften anbelangt. “10 Und: „Mit den heutigen Regeln kann die EU weder erweitert werden, noch ist sie zu notwendigen Entscheidungen befähigt." "11

Eine Strategie ,Zunächst vertiefen, um dann zu erweitern“ wirft allerdings erstens die grundsätzliche Frage auf, welche Projekte und Leitbilder geeignet sein könnten, als Basis für

8 Europäische Kommission: Erweiterungsstrategie und wichtigste Herausforderungen für den Zeitraum 20062007, KOM(2006) 649 endgültig, S. 6, abrufbar unter: http://ec.europa.eu/enlargement/pdf/key_documents/ 2006/Nov/com_649_strategy_paper_de.pdf (letzter Zugriff: 03.02.2007).

9 Dauderstädt/Lippert/Maurer: Die deutsche EU-Ratspräsidentschaft 2007, S. 28.

10 Angela Merkel: Rede anlässlich der Eröffnung des Internationalen Bertelsmann Forums, ,Die Zukunft der Europäischen Union“, 22.09.2006, Berlin, abrufbar unter: http://www.bundeskanzlerin.de/nn_5296/Content/DE/ Rede/2006/09/2006-09-23-bertelsmann.html (letzter Zugriff: 07.02.2007).

11 Angela Merkel: Rede vor dem Europäischen Parlament, 17.01.2007, Straßburg, abrufbar unter: http:// www.bundeskanzlerin.de/nn_5296/Content/DE/Rede/2007/01/2007-01-17-bkin-rede-ep.html (letzter Zugriff: 07.02.2007). 
eine Fortsetzung der Vertiefung der Integration zu dienen: „Nous devons poursuivre dans les mois qui viennent afin de préciser ce que l'on entend par,capacité d' absorption “ - expression qu'il faudrait d'ailleurs, à mon sens, modifier parce qu'il ne s'agit pas d'absorber tel ou tel mais de savoir quel projet les Etats européens veulent mener en commun."12

Die Schwierigkeiten bei der Suche nach geeigneten gemeinsamen Projekten für eine weitere Vertiefung der europäischen Integration sind bereits an anderer Stelle diskutiert worden. ${ }^{13}$ Angesichts der grundsätzlich unterschiedlichen Positionen der Mitgliedstaaten, von denen die einen für eine pragmatisch-funktionalistische Vorgehensweise plädieren, die auf konkrete Angebote und Vertiefungsprojekte in der policy- und politics-Dimension abzielt, während die anderen zunächst eine grundlegende institutionelle Reform der Europäischen Union in der polity-Dimension für unerlässlich halten, stellt sich zum einen die Frage nach der politischen Führung und zum anderen nach der Definition von Prioritäten in der Vertiefungsdiskussion.

Zweifelsohne werden sich mittelfristig im Rahmen einer konsensualen Paketlösung auf allen drei Ebenen Reform- und Vertiefungsprojekte definieren lassen. So werden in der policies-Dimension gegenwärtig bereits die Energie- und die Energieaußenpolitik der Union sowie der Klimaschutz als zentrale Projekte der deutschen EU-Ratspräsidentschaft diskutiert. Auch in den Bereichen Forschung und Entwicklung, Steuerpolitik, polizeiliche Zusammenarbeit oder Harmonisierung der Strafgesetzgebung sind weitere Schritte auf europäischer Ebene denkbar.

Zwei Punkte gilt es hierbei jedoch zu berücksichtigen: Erstens sollte die Europäische Union nach dem vorläufigen Scheitern des Verfassungsvertrages darum bemüht sein, durch eine effiziente, problemlösungsorientierte Politik greifbare Resultate zu liefern. ${ }^{14}$ Ein ,großer Wurf', das heißt ein großes neues gemeinsames Projekt (wie zum Beispiel der Aufbau einer gemeinsamen europäischen Verteidigung) zeichnet sich dabei in den kommenden Jahren jedoch nicht ab. ${ }^{15}$ Vielmehr sind detaillierte Reform- und Weiterentwicklungsvorschläge für einzelne Bereiche zu erwarten, deren Diskussion eine genaue Kenntnis europäischer Politiken und Rahmenbedingungen voraussetzt. Derartige Reformvorschläge stehen damit vor dem Problem, dass sie grundsätzlich schwer öffentlich vermittelbar sind. Die Union sollte daher versuchen, Erfolg versprechende Optionen europäischer Politikgestaltung frühzeitig in den öffentlichen Diskurs einzubringen, um auf diesem Weg das Verständnis für die Funktionsweise europäischer Politik zu verbessern und so an Legitimität zu gewinnen.

Zweitens wird ein gemeinsames, einheitliches Voranschreiten aller 27 Mitgliedstaaten in der policy-Dimension zukünftig eher die Ausnahme und nicht den Regelfall darstellen. Anstelle eines europäischen Gleichschritts ist daher zunehmend mit ad hoc Flexibilisierungsund Differenzierungsvarianten bei weiteren Vertiefungsprojekten zu rechnen, um denjenigen Mitgliedstaaten, die an einer Zusammenarbeit interessiert sind, Möglichkeiten hierzu auch innerhalb des Vertragsrahmens zu eröffnen. ${ }^{16} \mathrm{Um}$ ein Auseinanderdriften der EU$27(+)$ zu verhindern, muss allerdings auf der Ebene der politics-Dimension, das heißt insbe-

12 Catherine Colonna, französische Europaministerin, in einer Rede in Paris am 29.08.2006, abrufbar unter: http:/ /www.diplomatie.gouv.fr/fr/article-imprim.php3?id_article=40427 (letzter Zugriff: 07.02.2007).

13 Vgl. Anne Faber/Wolfgang Wessels: Strategien und institutionelle Perspektiven nach der Verfassungskrise: ,Funktionalistische' und ,institutionalistische' Wege zu einem neuen europäischen Verhandlungspaket, in: Politische Vierteljahresschrift 2/2006, S. 252-263.

14 Eine solche Strategie vertritt insbesondere auch die Kommission. Vgl. José Manuel Barroso, interviewt von Joachim Hesse: Where to Go From Here? The Commission's Perspective on the Future European Development, in: Zeitschrift für Staats- und Europawissenschaften 3/2005, S. 650-661, hier S. 652.

15 Vgl. auch Institut für Europäische Politik (Hrsg.): EU-25 Watch No. 3; Juli 2006, Berlin; Institut für Europäische Politik (Hrsg.): EU-25/27 Watch No. 4, Januar 2007, Berlin. 
sondere im Bereich der Entscheidungsverfahren, ein einheitlicher institutioneller Rahmen gewahrt bleiben, der die Grundregeln der Zusammenarbeit festlegt. Hier sind Reformen und Vertiefungsschritte wie die Einführung der doppelten Mehrheit oder die Einigung auf neue Flexibilisierungskonzepte denkbar, selbst wenn die angestrebte Ratifikation und Implementation des Verfassungsvertrages in allen Mitgliedstaaten von der deutschen Ratspräsidentschaft im Juni 2007 als mittelfristig nicht realisierbares Projekt eingestuft werden sollte. Eine der Lehren aus den gescheiterten Referenden zum europäischen Verfassungsvertrag ist, dass Fragen der institutionellen Architektur der Europäischen Union auf wenig Interesse bei den Bevölkerungen der Mitgliedstaaten stoßen und daher nicht für Referenden geeignet sind. Die zur Abstimmung gestellten Inhalte sind zu technisch und komplex, um als solche politisch mobilisierend wirken und als Gegenstände einer europaweiten Abstimmung fungieren zu können.

So paradox dies auf den ersten Blick klingen mag, müssen sich Befürworter der Strategie ,Erst vertiefen, um dann zu erweitern“ darüber hinaus fragen lassen, ob sich eine Vertiefung der Europäischen Union überhaupt losgelöst von zukünftigen Erweiterungen realisieren lassen wird. Die bisherige Entwicklung des Integrationsprozesses zeigt seit den 1970er Jahren einen engen zeitlichen und inhaltlichen Zusammenhang zwischen Erweiterungs- und Vertiefungsrunden. So wurde die Erweiterungsrunde um Großbritannien, Dänemark und Irland vorbereitet und begleitet von den Gipfeln in Den Haag (1969) und in Paris (1972), auf denen eine Reihe von zentralen Beschlüssen zur Vertiefung der politischen Zusammenarbeit in der Europäischen Union getroffen wurden. ${ }^{17}$ Auch wenn diese Beschlüsse erst mit großer zeitlicher Verzögerung in konkrete Resultate und institutionelle Weiterentwicklungen umgesetzt wurden, so war damit doch ein Muster etabliert, das im Rahmen der folgenden Erweiterungsrunden immer deutlicher zutage tritt. So wurde die Süderweiterung der Europäischen Gemeinschaft um Griechenland (1981), Spanien und Portugal (1986) begleitet von den Verhandlungen um die Einheitliche Europäische Akte (1986) und das Projekt eines einheitlichen Europäischen Binnenmarktes. Die sogenannte EFTA-Erweiterung um Schweden, Finnland und Österreich (1995) wiederum folgte auf den Vertrag von Maastricht, der die Wirtschafts- und Währungsunion etabliert hatte, und wurde begleitet von den Verhandlungen zum Vertrag von Amsterdam, der weitere Vertiefungsschritte im sozial- und arbeitsmarktpolitischen Bereich festschreiben sollte. Die Osterweiterung um $10+2$ neue Mitgliedstaaten (2004/07) schließlich sollte nicht nur durch den vielfach als unzureichend bewerteten ${ }^{18}$ Vertrag von Nizza (2000), sondern durch den Abschluss eines neuen Grundlagenvertrages, den Europäischen Verfassungsvertrag, abgesichert werden.

Vertiefungsprojekte mit weitreichenden Konsequenzen wurden somit auf europäischen Gipfeln in der Regel als ,Paketlösungen“ parallel zu Erweiterungsrunden ausgehandelt und

16 Für eine skeptische Beurteilung dieser Perspektive vgl. Sieglinde Gstöhl: Die Flexibilität der Europäischen Union gegenüber Mitgliedern und europäischen Drittstaaten, in: Jörg Glombowski u.a. (Hrsg.): Erweiterung und Integration der EU: Eine Rechnung mit vielen Unbekannten, Wiesbaden 2004, S. 147-173.

17 So wurde 1969 auf dem Gipfel von Den Haag die Einführung der Wirtschafts- und Währungsunion (WWU) und der Ausbau der Kompetenzen des Europäischen Parlaments sowie dessen Direktwahl ins Auge gefasst. Der Pariser Gipfel im Oktober 1972 führte zu dem Entschluss, die Europäische Gemeinschaft in eine ,Europäische Union " umzuwandeln. Darüber hinaus legten die Staats- und Regierungschefs einen neuen Zeitplan für den Beginn der Wirtschafts- und Währungsunion und neue Aufgabenfelder der Gemeinschaft in der Regional-, Umwelt-, Sozial-, Energie- und Industriepolitik fest.

18 Vgl. exemplarisch Klaus Hänsch: Maximum des Erreichbaren - Minimum des Notwendigen ? Die Ergebnisse von Nizza, in: integration 2/2001, S. 94-101, hier S. 94; Barbara Lippert: Neue Zuversicht und alte Zweifel: die Europäische Union nach ,Nizza“ und vor der Erweiterung, in: integration 2/2001, S. 179-193, hier S. 179 und S. 182-183; Andreas Maurer: Entscheidungseffizienz und Handlungsfähigkeit nach Nizza: die neuen Anwendungsfelder für Mehrheitsentscheidungen, in: integration 2/2001, S. 133-145, hier S.141. 
markierten dabei stets die Kompromisslinie zwischen Befürwortern einer Erweiterung und Befürwortern einer engeren wirtschaftlichen und politischen Zusammenarbeit: „This has been achieved by preceding, or at least accompanying, each enlargement round since the Mediterranean round with treaty reforms that have had as at least part of their purpose advancing institutional and policy deepening before they can be threatened by widening. [...] Widening has thus been an important factor in driving deepening. "19

Aufgrund der heterogenen Präferenzen der Mitgliedstaaten in der Vertiefungs- und Erweiterungsfrage ist nicht anzunehmen, dass substanzielle Vertiefungen oder weitreichende Reformen des institutionellen Rahmens ohne den (tatsächlichen oder angenommenen) Reformdruck zustande kommen werden, den Vertiefungsbefürworter - neben dem Verweis auf globale Trends und Veränderungen und die daraus resultierenden Anpassungserfordernisse der EU - nicht zuletzt auch aus einer erneuten Erweiterungsrunde ableiten können. Vor diesem Hintergrund erscheint eine Strategie, die ausschließlich oder vorrangig auf eine Vertiefung der Europäischen Union setzt, wenig Erfolg versprechend. Gegenwärtig tritt eine solche Strategie zwar stärker in den Vordergrund, da sie nach dem vorläufigen Scheitern des Verfassungsvertrages durch das implizite Argument der ,nachholenden Vertiefung ' gestärkt wird und sich auf eine zunehmende Erweiterungsskepsis in den alten Mitgliedstaaten der Europäischen Union berufen kann. Sollte sich in den nächsten ein bis zwei Jahren jedoch der Eindruck verstärken (und wissenschaftlich belegen lassen), dass die Osterweiterung keinen beziehungsweise zumindest nicht den vielfach prognostizierten disruptiven, qualitativen Wandel der bestehenden Strukturen und Entscheidungsmechanismen der Europäischen Union zur Folge gehabt hat, so verliert die Strategie der vorrangigen Vertiefung zur Kompensation der Osterweiterung massiv an Glaubwürdigkeit.

\section{Zunächst erweitern, um dann zu vertiefen}

Das Kernkonzept einer Strategie ,Zunächst erweitern, um dann zu vertiefen ' beinhaltet spiegelbildlich zu den eben diskutierten Punkten seine eigenen Vor- und Nachteile sowie offene Fragen. Vertreter einer solchen Strategie verweisen zum einen auf die globale Rolle der Europäischen Union als Sicherheits- und Stabilitätsanker auf dem europäischen Kontinent und ihre daraus erwachsende Verantwortung für eine integrative Politik: Nur die Beitrittsperspektive biete jungen Demokratien und Marktwirtschaften den sicheren Rahmen, in dem sie weiter stabilisiert werden könnten, und nur auf diesem Weg ließe sich für die Europäische Union ein verlässliches politisches Umfeld etablieren. Zum anderen dürfe die Europäische Union die Chancen, die jede weitere Erweiterung biete, nicht verpassen, wenn sie im globalen Wettbewerb mit Asien und anderen Wirtschaftsräumen nicht erheblich an Attraktivität und Einfluss verlieren wolle: „Enlargement is the only option if we want Europe to remain a leading economic force on the international stage." 20 Auch der britische Premierminister Tony Blair betonte in seiner Antrittsrede zur EU-Ratspräsidentschaft im Juni 2005 diese beiden Punkte: „Such a Europe - its economy in the process of being modernised, its security enhanced by clear action within our borders and beyond - would be a confident Europe. It would be a Europe confident enough to see enlargement not as a threat, as if membership were a zero sum game in which old members lose as new members gain, but an ex-

19 Neill Nugent: Distinctive and Recurring Features of Enlargement Rounds, in: Neill Nugent (Hrsg.): European Union Enlargement, Houndmills/Basingstoke 2004, S. 64.

20 Geoff Hoon: 50 Years on - the EU in a Globalised World, Rede in Dublin am 20.11.2006, abrufbar unter: http://www.fco.gov.uk/servlet/Front?pagename=OpenMarket/Xcelerate/ ShowPage\&c=Page\&cid=1140686158923\&a=KArticle\&aid=1163674254957 (letzter Zugriff: 07.02.2006). 
traordinary, historic opportunity to build a greater and more powerful union. Because be under no illusion: if we stop enlargement or shut out its natural consequences, it wouldn't, in the end, save one job, keep one firm in business, prevent one delocalisation. “21 Die Europäische Union könne und müsse, so die Argumentation, beitrittswillige und -fähige Staaten aus diesen beiden Gründen daher aufnehmen, ohne neue Hürden zu errichten. Fundamentale institutionelle Reformen oder politische Vertiefungsschritte seien dafür nicht erforderlich: ${ }^{22}$ „But none of this has required a EU Constitution! Enlargement is working fine without it. “23

$\mathrm{Zu}$ den Vertretern einer solchen Strategie zählen neben dem Vereinigten Königreich insbesondere Finnland ${ }^{24}$ und Irland. Die Position des Vereinigten Königreiches geht, betrachtet man einzelne Äußerungen, sogar in Richtung einer Strategie des ,Erweiterns, nicht aber Vertiefens'. Hinter dieser Position steht nicht zuletzt die Hoffnung, dass die Europäische Union, je größer und heterogener sie wird, institutionelle und politische Vertiefungsbestrebungen zunehmend unmöglich machen wird und die Debatte um das ungeliebte Leitbild der ,Vereinigten Staaten von Europa' damit ein für allemal beenden könnte. ${ }^{25}$

Offen bleibt im Rahmen einer Strategie ,Zunächst erweitern, um dann zu vertiefen“ jedoch die Frage, ob tatsächlich negative quantitative und qualitative Rückkoppelungseffekte von Erweiterungsrunden auf Vertiefungsprojekte ausgehen, und wenn ja, wie stark diese jeweils ausgeprägt sind. Eine reine Zunahme der Zahl von Mitgliedstaaten ist in jedem Fall nicht automatisch gleichbedeutend mit einem breiteren, heterogeneren Interessen- und Präferenzspektrum, sondern kann sich auch in einer Betonung und Stärkung bestimmter Positionen und Präferenzen ausdrücken, die unter den alten Mitgliedstaaten bereits bestanden haben. ${ }^{26}$ Generell scheint zu gelten, dass Erweiterungen keine big bangs in der Entwicklung der Europäischen Union darstellen, sondern bestehende Dynamiken und Projekte in einer pfadabhängigen und subtileren Art und Weise beeinflussen und neu gewichten. Erweiterungen lassen sich daher nicht als, elegante' Methode zur Verhinderung einer Vertiefung der Union einsetzen, sondern können, wie im vorangegangenen Punkt bereits angesprochen, in Abhängigkeit von den Interessen der neuen Mitgliedstaaten sowie den Entscheidungsverfah-

21 Tony Blair: Rede vor dem Europäischen Parlament am 23.06.2005, abrufbar unter: http://www.britischeboschaft.de/en/news/items/050623.htm (letzter Zugriff: 31.01.2007).

22 Vgl. hierzu die Äußerung von Margaret Beckett: „But [...] changes will have to be far more modest than those outlined by Mr Barroso", zitiert in der online Ausgabe des Guardian, 18.10.2006, abrufbar unter: http:// www.guardian.co.uk/eu/story/0,,1924810,00.html (letzter Zugriff: 07.02.2007).

23 Charles Tannock: Enlargement is working without an EU Constitution, Rede am 05.10.2004, abrufbar unter: http://www.conservatives.com/tile.do?def=news.story.page\&obj_id=116510\&speeches=1\# (letzter Zugriff: 07.02.2007).

24 Die erweiterungsfreundliche Position der finnischen Regierung schlug sich auch in der finnischen Ratspräsidentschaft im zweiten Halbjahr 2006 nieder. Matti Vanhanen bilanzierte in einer Rede zum Ende der Ratspräsidentschaft: „Finnland hatte es sich als ein Ziel für seine Präsidentschaft gesetzt, dass die Erweiterungspolitik der Union offen bleibt und dass wir verdienten Anwärtern nicht die Tür versperren. [...] Um die Union dürfen keine künstlichen Grenzen gezogen werden." Matti Vanhanen: Rede vor dem Europäischen Parlament am 18.12.2006, abrufbar unter: http://eu2006.navigo.fi/news_and_documents/speeches/vko51/en_GB/178743/ (letzter Zugriff: 06.02.2007).

25 „The essence of the case that widening threatens deepening is that the larger, the more diverse, and the less cohesive the EU becomes then so does decision-making become more difficult and, therefore, policy development more problematical. All member states have recognized that there is at least something in this case, whilst the UK, for long the member state least willing to support integrationist advance, has also hoped there is much in the case". Nugent: Distinctive and Recurring Features of Enlargement Rounds, S. 64; Hervorhebung im Original.

26 So wird z.B. die Osterweiterung der EU oft als Stärkung der ,Atlantiker ' in der EU gewertet, die eine enge Koppelung der Gemeinsamen Außen- und Sicherheitspolitik (GASP) an die Außenpolitik der USA vertreten. Vgl. hierzu Michael Baun: Intergovernmental Politics, in: Neill Nugent: European Union Enlargement, S. 132145, hier S. 138-140. 
ren in der Union ${ }^{27}$ im Gegenteil ebenso gut zu einer Intensivierung des Vertiefungsdiskurses und zur Begründung neuer gemeinsamer Politiken führen.

Darüber hinaus ist die Strategie ,Zunächst vertiefen, um dann zu erweitern' nicht losgelöst von der Frage einer Grenzziehung für die Europäische Union diskutierbar. Zwar suggeriert diese Strategie eine nahezu unbegrenzte Erweiterbarkeit der Union; ${ }^{28}$ in der politischen Debatte wird die Erweiterungsfrage seit der Osterweiterung 2004/2007 jedoch immer häufiger an die Frage nach der geografischen finalité der Union rückgebunden. Die Erweiterung der Europäischen Union ad infinitum, so die Befürchtung vieler, würde zu einer Union mit ,schwammigen Grenzen' nach außen und einer rechtlich noch differenzierteren Mitgliedschaft nach innen führen, was für ihren Zusammenhalt wie auch für ihre Funktionsfähigkeit gravierende Folgen hätte. Auch die deutsche Bundeskanzlerin hat mehrfach festgestellt, dass die Europäische Union ihre Grenzen klar definieren müsse: „Europa muss seine Konturen nach innen und nach außen schärfen, denn ohne klare Verfasstheit über die geografische Ausdehnung wird Europa in einer sich rasant verändernden Welt schwer bestehen. ${ }^{‘ 29}$

Insbesondere in diesem Punkt drücken sich die bereits angesprochene ,Erweiterungsmüdigkeit" und die Sorge vieler Regierungen aus, dass es mittelfristig unter den Bevölkerungen der Mitgliedstaaten keine Mehrheiten für neuerliche Erweiterungsrunden geben wird. ${ }^{30}$ Dies ist angesichts von obligatorischen beziehungsweise fakultativen Referenden zu einer neuerlichen Erweiterung der Europäischen Union in einigen Mitgliedstaaten längst kein kosmetisches Problem mehr. Eine Vorgehensweise in der Erweiterungsfrage, die diese möglicherweise fehlende Legitimationsbasis zu übergehen versucht, steht darüber hinaus auch in klarem Widerspruch zur neuen Erweiterungsstrategie der Kommission und ihrem dritten Schwerpunkt auf „Kommunikation“. ${ }^{31}$ Selbst wenn es zum gegenwärtigen Zeitpunkt wenig sinnvoll und realistisch erscheint, die endgültigen geografischen Grenzen der Europäischen Union zu einem Zeitpunkt festlegen (und damit bestimmte Staaten kategorisch von einer Beitrittsperspektive ausschließen) zu wollen, zu dem die Natur dieses, objet politique noch immer nicht definiert ist, so ist in den kommenden Jahren eine sehr viel klarere und direktere Vermittlung der Erweiterungsstrategie der Europäischen Union in den Mitgliedstaaten in der Tat unerlässlich. Eine solche Kommunikation muss unmissverständlich und nachhaltig deutlich machen

- welche neuen Staaten eine realistische Beitrittsperspektive haben (wer)

27 Vgl. hierzu Bernard Steunenberg: Enlargement and Institutional Reform in the European Union: Separate or Connected Issues?, in: Constitutional Political Economy 12/2001, S. 351-370.

28 Seit dem 3. Oktober 2005 laufen die Beitrittsverhandlungen mit Kroatien und der Türkei. Die ehemalige jugoslawische Republik Mazedonien hat seit Dezember 2005 den Status eines Beitrittslandes, es wurden jedoch bislang noch keine Beitrittsverhandlungen aufgenommen. Die anderen Länder des westlichen Balkans werden von der Europäischen Union als potenzielle Bewerber angesehen, deren Mitgliedschaft langfristig bei Erfüllung der Beitrittskriterien erwünscht ist. Hierzu zählen: Albanien, Bosnien und Herzegowina, Montenegro und Serbien. Auch wenn sich die Beziehungen zwischen der Europäischen Union und der Ukraine in einem Prozess stetiger Intensivierung befinden, wird der Ukraine bislang keine Beitrittsperspektive in Aussicht gestellt.

29 Angela Merkel: Rede anlässlich der Eröffnung des Internationalen Bertelsmann Forums „Die Zukunft der Europäischen Union“, Berlin, 22. September 2006.

30 Laut dem Standard Eurobarometer für Herbst 2006 sprachen sich 46 Prozent der Unionsbürger für, 42 Prozent gegen künftige Erweiterungen aus. Quelle: Pressemitteilung zum Standard Eurobarometer 66: Herbst 2006, abrufbar unter http://europa.eu/rapid/pressReleasesAction.do?reference=IP/06/1825\&format=HTML\&aged= $0 \&$ language $=E N \&$ guiLanguage $=$ en (letzter Zugriff: 5.2.2007). Damit sinkt der Anteil derjenigen, die einer zukünftigen Erweiterung zustimmen, seit 2004 kontinuierlich, während der Anteil der Erweiterungsgegner ebenso kontinuierlich steigt. Vgl. hierzu auch Dauderstädt/Lippert/Maurer: Die deutsche EU-Ratspräsidentschaft 2007, S. 27

31 Europäische Kommission: Enlargement Strategy and main Challenges 2006 - 2007, S. 5. 
- aus welchen Gründen diese neuen Staaten in die Europäische Union aufgenommen werden sollen (warum)

- zu welchem voraussichtlichen Termin ein Beitritt erfolgen könnte (wann)

- unter welchen Bedingungen der Beitritt erfolgen wird (wie) und

- welche Schritte die Europäische Union in der Zwischenzeit unternehmen wird, um auf die Erweiterung vorbereitet zu sein.

Vor dem Hintergrund der diskutierten Punkte erscheint eine Strategie, die ausschließlich oder vorrangig auf eine Erweiterung setzt, mindestens ebenso zum Scheitern verurteilt wie eine Strategie, die ausschließlich eine Vertiefung der Europäischen Union anstrebt.

\section{Gleichzeitig erweitern und vertiefen}

Die Strategie des gleichzeitigen Erweiterns und Vertiefens repräsentiert im Kern den ,Königsweg', den die Europäische Gemeinschaft/Europäische Union bislang als Kompromisslösung zwischen den Vertretern der beiden oben diskutieren Strategien gewählt hat. So wurde jede der fünf Erweiterungsrunden der Europäischen Union von der Ausweitung der inhaltlichen Zuständigkeitsbereiche sowie einer institutionellen Vertiefung der Zusammenarbeit begleitet. Auch im Rahmen der letzten beiden Erweiterungsrunden 2004 und 2007 wurde diese Strategie durch die Verträge von Amsterdam und Nizza sowie die Einberufung des Verfassungskonvents forciert. Vertiefende Reformprojekte werden auch von der Europäischen Kommission als unumgängliche Voraussetzung für eine Fortsetzung des Erweiterungsprozesses gesehen. ${ }^{32}$ Allerdings liegt das letzte zentrale Reform- und Vertiefungsprojekt der Union, der Europäische Verfassungsvertrag, nach den gescheiterten Referenden in den Niederlanden und in Frankreich bereits seit Mitte $2005^{33}$ auf Eis.

Gerade die jüngste Erweiterungsrunde hat gezeigt, dass die traditionelle Strategie einer parallelen Erweiterung und Vertiefung zunehmend unter Druck gerät. Mitgliedstaaten, die eine vorrangige Vertiefung beziehungsweise eine vorrangige Erweiterung befürworten, blockieren sich gegenseitig bei der Umsetzung des jeweils ungeliebten Parts der Parallel-Strategie. Gegenwärtig scheint daraus sowohl im Hinblick auf eine weitere Vertiefung als auch im Hinblick auf künftige Erweiterungen der Europäischen Union eine ,fatigue zu resultieren, die sich exemplarisch an der selbst verordneten, seit Juni 2005 andauernden ,Denkpause“ festmachen lässt. Die Union verharrt auf dem Status quo, ohne dass eine Weiterentwicklung von Vertiefungs- oder Erweiterungsszenarien erkennbar würde. Der Bewegungsspielraum der Union in die eine oder die andere Richtung ist momentan offensichtlich äußerst klein; die Belastbarkeit der traditionellen Kompromissstrategie scheint an ihre Grenzen gelangt zu sein. Damit stellen sich fundamentale Fragen nach der Verfasstheit und den Zielen der Union zu Beginn des 21. Jahrhunderts: Ist eine Einigung über grundsätzliche Fragen endgültig unausweichlich, das heißt bedarf es nun erst einer gemeinsamen Zielbestimmung der Europäischen Union? Oder hat die Europäische Union mit 27 Mitgliedstaaten jene ,kritische Masse erreicht, mit der die Zeit beziehungsweise die Möglichkeit eines gemeinsamen, einheitlichen

32 Vgl. exemplarisch José Manuel Barroso: Seeing through the hallucinations: Britain and Europe in the 21st century, Hugo Young Lecture am 16.10.2006 in London, abrufbar unter http://europa.eu/rapid/pressReleasesAction.do?reference=SPEECH/06/602\&format=HTML\&aged=0\&language=EN\&guiLanguage=en, $\quad$ (letzter Zugriff: 7.2.2007).

33 Angesichts der gescheiterten Referenden wurde im Rahmen der Tagung des Europäischen Rates am 16./17. Juni 2005 in Brüssel von den Staats- und Regierungschefs eine , Reflexionsphase' anberaumt, die bis heute andauert. Vgl. Erklärung der Staats- und Regierungschefs der Mitgliedstaaten der Europäischen Union zur Ratifizierung des Vertrags über eine Verfassung für Europa, DOC/05/3, Brüssel 18.06.2005, abrufbar unter http:// europa.eu/rapid/pressReleasesAction.do?reference=DOC/05/3\&format=HTML\&aged=0 \&language=DE\&gui Language=en (letzter Zugriff: 6.2.2007). 
Integrationsfortschritts aller Mitgliedstaaten vorbei ist? Werden weitere Vertiefungs- und Erweiterungsprojekte also zukünftig unabhängig voneinander stattfinden, und Reformprojekte jeweils nur von kleineren Gruppen von Mitgliedstaaten vorangetrieben werden?

Zumindest eine Akzentverschiebung in der traditionellen Strategie ,Gleichzeitig erweitern und vertiefen ' lässt sich gegenwärtig konstatieren: Statt weiterhin gleichzeitig erweitern und vertiefen oder , nachholend ' vertiefen zu wollen, scheint die Mehrheit der Mitgliedstaaten spätestens nach der Erweiterung um Bulgarien und Rumänien zum 1. Januar 2007 zunächst eine Phase der Konsolidierung zu präferieren und verweist dabei verstärkt auf die

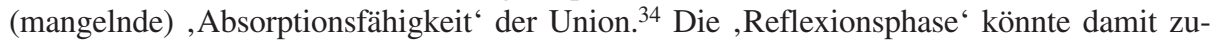
mindest für die kommenden fünf bis zehn Jahre zum Dauerzustand werden. Aus Sicht einiger Beobachter wäre genau dies als Beweis dafür anzusehen, dass die Europäische Union zufrieden stellend arbeitet und ein stabiles institutionelles Equilibrum erreicht hat: Demnach repräsentiert die Union nun ein ,reifes “ politisches System ,that does not need continually to move forward on a neo-functionalist bicycle in order to be stable. " ${ }^{35}$ Ohne neue Impulse und Projekte, die ein gemeinsames Voranschreiten ermöglichen, ist jedoch nach Ansicht anderer Beobachter die Stabilität der Union in Frage gestellt - sie befürchten ein Auseinanderbrechen der Europäischen Union in kleinere Staatengruppen. Ob die weitere Verzögerung einer Diskussion um die Zukunft der Erweiterungs- und Vertiefungsperspektiven der Europäischen Union zumindest bis zum Abschluss der deutschen Ratspräsidentschaft im Juni 2007 eine problemadäquate Strategie darstellt, kann zumindest angezweifelt werden.

\section{Ausblick: Leitlinien einer neuen Vertiefungs- und Erweiterungsstrategie für die EU-27}

Die vorangegangene Diskussion hat zwei Punkte verdeutlicht: Erstens, dass Erweiterung und Vertiefung sich in der bisherigen Entwicklung des Integrationsprozesses ebenso stark wechselseitig bedingt haben, wie sie sich momentan gegenseitig zu behindern scheinen. Der Satz ,Ohne Erweiterung keine Vertiefung, und ohne Vertiefung keine Erweiterung ' gilt daher ebenso wie der Satz ,Jede Erweiterung erhöht die Hürden für eine neuerliche Vertiefung, und jede Vertiefung erhöht die Hürden für neue Beitritte'. Dies ist jedoch nicht das Resultat , automatisch` ablaufender, endogener Prozessdynamiken, sondern zurückzuführen auf ganz konkrete, divergierende Interessen und Strategien der Mitgliedstaaten in der Vertiefungsund Erweiterungsfrage.

Zweitens sind alle drei klassischen Strategien, die auf europäischer Ebene die politische Vertiefungs- und Erweiterungsdebatte dominieren, nicht in der Lage, zu einer nachhaltigen und zukunftsorientierten Neuorientierung der EU-27 beizutragen. Eine Verzögerung politischer Grundsatzentscheidungen bietet keine tragfähige Basis für die unmittelbar anstehenden Aufgaben wie insbesondere die schnellstmögliche Beendigung der Verfassungskrise und der ,Reflexionsphase ‘, ${ }^{36}$ die Vorbereitung auf beziehungsweise die Fortführung der Beitrittsverhandlungen mit den Staaten des westlichen Balkans sowie die Fortführung der

34 Siehe dazu den Beitrag in diesem Heft von Kai-Olaf Lang und Daniela Schwarzer: Die Diskussion über die Aufnahmefähigkeit der EU - Plädoyer für eine neue Erweiterungsstrategie.

35 Andrew Moravcsik: What Can We Learn from the Collapse of the European Constitutional Project?, Politische Vierteljahresschrift 2/2006, S. 219-241, hier S. 237.

36 Zu den Perspektiven und Optionen der EU im Umgang mit dem gescheiterten Verfassungsvertrag vgl. eine Vielzahl von Beiträgen in der integration wie Udo Diedrichs/Wolfgang Wessels: Die Europäische Union in der Verfassungsfalle? Analysen, Entwicklungen und Optionen, in: integration 4/2005, S. 287-306; Daniel Thym: Weiche Konstitutionalisierung - Optionen der Umsetzung einzelner Reformschritte des Verfassungsvertrags ohne Vertragsänderung, in: integration 4/2005, S. 307-315; Daniel Göler/Hartmut Marhold: Die Zukunft der Verfassung Überlegungen zum Beginn der Reflexionsphase, in: integration 4/2005, S. 332-335; Daniel Göler/Mathias Jopp: Die europäische Verfassungskrise und die Strategie des ,langen Atems', in: integration 2/2006, S. 91-105. 
Beitrittsverhandlungen mit der Türkei. Auch für immer stärker auf die Tagesordnung der Union drängende Fragen wie die Energiesicherheit und die Energieaußenpolitik ist eine klare strategische Positionierung der Europäischen Union als außenpolitischem Akteur unerlässlich.

Daher muss in der Union jetzt mit der Suche nach neuen, differenzierten Strategien und Antworten auf die Vertiefungs- und Erweiterungsdebatte begonnen werden. Dabei ist zu beachten, dass die Europäische Union in eine Vielzahl von politischen Verpflichtungen und pfadabhängigen Entwicklungen eingebunden ist; ein radikaler Bruch mit traditionellen Konzepten ist daher höchst unwahrscheinlich. Grundsätze und Leitideen einer neuen Strategie, die sich in die bestehenden Konzepte durchaus integrieren ließen, sind in den vorangegangenen Punkten jedoch bereits mehrfach angeklungen.

Im Hinblick auf zukünftige Erweiterungen sind drei Anforderungen an eine neue Strategie festzuhalten:

- Differenzierung der Vorgehensweise und Angebote gegenüber Drittstaaten, um einen ,Beitrittsautomatismus" und einen unbegrenzten Erweiterungsdruck auf die Union zu verhindern. ${ }^{37}$

- Transparenz in der Vorgehensweise gegenüber Drittstaaten und ihrer Einbeziehung in den institutionellen Aufbau der Union sowie Vermeidung von reaktiven ad-hoc-Angeboten. Stattdessen ist die aktive Definition neuer ,europapolitischer Nachbarschaftsräume " und klarer strategischer Ziele und Leitlinien der Europäischen Union für diese Räume anzustreben, die auf den Kopenhagener Kriterien aufbauen.

- Kommunikation der Inhalte und Ziele der Erweiterungs- und Assoziierungsstrategie in den Mitgliedstaaten der Europäischen Union, um eine breitere Legitimationsbasis zu schaffen.

Damit stellt sich verstärkt auch die Frage nach Alternativen zu einer Erweiterung, und zwar sowohl aus Perspektive der Union selbst als auch aus Sicht derjenigen Drittstaaten, denen keine Beitrittsperspektive angeboten werden kann beziehungsweise soll. Hierzu sind in den letzten Jahren eine Reihe von Vorschlägen gemacht worden, die insbesondere auf eine Intensivierung der europäischen Nachbarschaftspolitik abzielen. ${ }^{38}$ Barbara Lippert hat in diesem Zusammenhang für „Assoziierungsabkommen neuen Typs“39 mit den Staaten an der neuen Ostgrenze der Europäischen Union ${ }^{40}$ plädiert, in deren Fällen sich die Union bereits mehr oder minder deutlich gegen einen Beitritt ausgesprochen hat. ${ }^{41}$ Solche Assoziierungsabkommen könnten in den Bereichen Wirtschaft, Politischer Dialog, außen- und sicherheitspolitische Kooperation, Innere Sicherheit und Hilfe differenzierte Instrumente und Maßnahmen zur Unterstützung von Drittstaaten vorsehen, sie systematisch in den Meinungsbildungsprozess der Europäischen Union einbeziehen und so eine „Modernisierungs- und Stabilitätspartnerschaft“ begründen. ${ }^{42}$ Bewährt sich eine solche Strategie, könnte daraus der Aufbau eines Europas der konzentrischen Kreise in unterschiedlichen funktionalen Bereichen resultieren, das heißt eine ,gesamteuropäische Aufgabenkonföderation“. ${ }^{43}$ Darüber hinaus ist die Europäische Union aber auch aufgefordert, über die zukünftige Ge-

37 Vgl. auch Geoffrey Harris: The Wider Europe, in: Fraser Cameron (Hrsg.): The future of Europe. Integration and enlargement, New York 2004, S. 98-113, hier S. 99.

38 Vgl. Barbara Lippert: Assoziierung plus gesamteuropäische Aufgabenkonföderation: Plädoyer für eine selbstbewusste EU-Nachbarschaftspolitik, in: integration 2/2006, S. 149-157; Harris: The Wider Europe, S. 98-113.

39 Lippert: Assoziierung plus gesamteuropäische Aufgabenkonföderation, S. 150.

40 Dies betrifft insbesondere die Ukraine, Moldau und Georgien, Belarus, Armenien und Aserbaidschan.

41 Vgl. Harris: The Wider Europe, S. 108.

42 Lippert: Assoziierung plus gesamteuropäische Aufgabenkonföderation, S. 152.

43 Vgl. hierzu ausführlich Lippert: Assoziierung plus gesamteuropäische Aufgabenkonföderation, S. 153-156. 
staltung ihrer Beziehungen zum Mittelmeerraum und zu Staaten wie Algerien, Marokko, Ägypten, Israel, Tunesien und anderen nachzudenken. ${ }^{44}$

In der Tat scheint es keine Alternative zur Stärkung flexibler Instrumente und Kooperationsformen zu geben, auch wenn eine solche Strategie einen Kurswechsel der Europäischen Union in ihrem Auftreten gegenüber den genannten Staaten sowie eine klare Definition (und Umsetzung) attraktiver Angebote voraussetzt. Soll die Etablierung einer solchen ,selbstbewusste[n] EU-Nachbarschaftspolitik“" 45 gelingen, so muss die Union zudem in den nächsten Jahren auf eine Reihe von Fragen Antworten finden: In der Innenperspektive muss eine Trennschärfe zwischen Assoziierung und Mitgliedschaft erhalten bleiben, um zu verhindern, dass durch eine immer engere Assoziierung, faktische "Vollmitgliedschaften entstehen. Hier stellt sich vor allem die Frage, wie eine institutionelle Einbeziehung assoziierter Staaten in Meinungsbildungs-, nicht aber in Entscheidungsprozesse auf die Dauer durchzuhalten und zu rechtfertigen wäre. Darüber hinaus würde eine enge Assoziierung von Drittstaaten sehr wahrscheinlich zur Schaffung von schwer kommunizierbaren ,fuzzy borders' führen, das heißt zu einer erneut deutlich intransparenteren Europäischen Union. In der Folge muss mit einer weiteren Schwächung der Legitimationsbasis in den Mitgliedstaaten gerechnet werden. In der Außenperspektive hingegen muss die Europäische Union klare Optionen aufzeigen und Angebote machen, die für Drittstaaten eine enge Kooperation auch ohne Beitrittsperspektive attraktiv machen. Dass dies keine leichte Aufgabe sein wird, zeigen alle bisherigen Reaktionen seitens der in Frage kommenden Staaten: „Spokesmen for the Ukraine have echoed the concern that 'neighbourhood" is really a polite term for exclusion. "46 Die aufgeführten Punkte verdeutlichen, dass auch für eine Strategie, die auf Assoziierung anstelle neuer Beitrittsrunden setzt, die Klärung des Selbstverständnisses der Europäischen Union in Grundsatzfragen eine unverzichtbare Voraussetzung darstellt. ${ }^{47}$

Hinsichtlich weiterer Vertiefungsschritte sollten als Grundsätze und Leitideen einer neuen Strategie gelten:

- Flexibilisierung der Europäischen Union in der policy-Dimension, das heißt ein explizites Bekenntnis zu flexiblen Formen der Zusammenarbeit in kleineren Gruppen von Mitgliedstaaten, die für andere Mitgliedstaaten offen bleiben und als Avantgardes fungieren können.

- Stabilisierung des einheitlichen institutionellen Rahmens der Europäischen Union auf der Basis des Status quo, das heißt Vermeidung eines Europa à la carte in der politics- und in der polity-Dimension.

- Kommunikation und Diskussion konkreter politischer Vertiefungs- und Reformprojekte in den Mitgliedstaaten, um eine breitere Akzeptanz und Legitimationsbasis für europapolitische Themen zu schaffen und zur Herausbildung einer europäischen Öffentlichkeit beizutragen.

Aufbauend auf diesen Prinzipien könnte eine neue Strategie entwickelt werden, die Vertiefung und Erweiterung schlüssig und nachhaltig miteinander verbindet. Dabei werden jeder (Neu-)Positionierung, die die Union in der Frage ,Vertiefung und/oder Erweiterung? vornehmen wird, politische Grundsatzentscheidungen über ihr Selbstverständnis, ihre Ziele und ihre geopolitische Rolle vorangehen müssen. Es liegt daher in der Hand der Mitgliedstaaten, durch eine konstruktive, offene Debatte über die Ziele und die zukünftige Gestalt

44 Vgl. ausführlich Harris: The Wider Europe, S. 101-108.

45 Lippert: Assoziierung plus gesamteuropäische Aufgabenkonföderation, S. 149.

46 Harris: The Wider Europe, S. 110.

47 Vgl. hierzu auch Lippert: Assoziierung plus gesamteuropäische Aufgabenkonföderation, S. 156-157. 
der Union dazu beizutragen, dass Vertiefung und Erweiterung, statt endgültig zu einem Dilemma zu werden, wieder zu den beiden Hauptantriebskräften für die Weiterentwicklung der Integration werden. Die Zustimmung der Bevölkerung in den Mitgliedstaaten zu einer neuen Vertiefungs- und Erweiterungsstrategie wird dabei ebenso entscheidend sein wie die Präferenzen der mitgliedstaatlichen Regierungen als den zentralen Akteuren: „One thing is sure, however, the widening-deepening debate will continue for many years to come.“48

Der Beitrag ist hervorgegangen aus EU-CONSENT, einem europaweiten Forschernetzwerk zu den Auswirkungen von Vertiefung und Erweiterung der EU im Rahmen des 6. Forschungsrahmenprogramms der Europäischen Union, das vom Jean Monnet Lehrstuhl von Prof. W. Wessels an der Universität zu Köln koordiniert wird.

48 Fraser Cameron: Europe's future, in: Fraser Cameron (Hrsg.): The future of Europe. Integration and enlargement, S. 149-161, hier S. 161. 